New Generation Computing, 20(2002)1-2

Ohmsha, Ltd. and Springer-Verlag

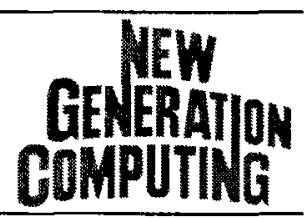

(C)Ohmsha, Ltd. 2002

\title{
Preface
}

\section{Partial Evaluation and Program Transformation Day}

\author{
Robert GLÜCK \\ Waseda University \\ 3-4-1 Okubo, Shinjuku-ku, Tokyo 169-8555 \\ PRESTO, JST\& Institute for Software Production Technology \\ glueck@acm.org \\ Yoshihiko FUTAMURA \\ Dept. of Information and Computer Science, \\ Waseda University \\ 3-4-1 Okubo, Shinjuku-ku, Tokyo 169-8555 \\ futamura@futamura.info.waseda.ac.jp
}

This special issue of New Generation Computing contains five, fully reviewed papers presented at the Partial Evaluation and Program Transformation Day held at Waseda University, Tokyo, November 15, 1999. This one-day colloquium featured talks and discussions on semantics-based program transformation with an emphasis on program specialization, generalized partial computation, and automatic program transformation.

The contributions in this special issue represent state-of-the-art research ranging from theoretical works to practical studies, and across different programming language paradigms (functional, logic, object-oriented). They are arranged alphabetically. Here is a brief summary of the contributions:

- Elvira Albert and Germán Vidal. The Narrowing-Driven Approach to Functional Logic Program Specialization. Surveys the state-of-the-art of narrowingdriven partial evaluation of functional-logic programs.

- Kenichi Asai. Binding-Time Analysis for Both Static and Dynamic Expressions. Presents a novel binding-time analyzer for a functional language and proves the correctness of the constraint solving algorithm.

- Olivier Danvy, Bernd Grobauer, and Morten Rhiger. A Unifying Approach to Goal-Directed Evaluation. Addresses the challenge of implementing goaldirected evaluation using computational monads and partial evaluation.

- Yoshihiko Futamura, Zenjiro Konishi, and Robert Glück. Automatic Program Transformation System Based on Generalized Partial Computation. Discusses the power of a program transformation system utilizing a theorem prover and a partial evaluator.

- Hidehiko Masuhara and Akinori Yonezawa. A Portable Approach to Dynamic 
Optimization in Run-time Specialization. Proposes a run-time bytecode specialization technique for the Java virtual machine language.

We would like to thank all authors, the external reviewers, and the journal editors for providing their expertise and excellent contributions. 